\title{
Non-weight-bearing short knee radiographs to evaluate coronal alignment before total knee arthroplasty
}

\author{
Sheng Pan ${ }^{1}$, Chaoran Huang ${ }^{1}$, Xingchen Zhang ${ }^{1}$, Ruxin Ruan ${ }^{1}$, Ziwen Yan ${ }^{1}$, Zheng Li ${ }^{1}$, Yong Pang ${ }^{1}$, \\ Kaijin Guo ${ }^{1}$, Xin Zheng ${ }^{1,2}$ \\ ${ }^{1}$ Department of Orthopedics, The Affiliated Hospital of Xuzhou Medical University, Xuzhou, China; ${ }^{2}$ Department of Orthopedics, Zhujiang \\ Hospital of Southern Medical University, Guangzhou, China
}

Contributions: (I) Conception and design: S Pan, K Guo, X Zheng; (II) Administrative support: K Guo, X Zheng; (III) Provision of study materials or patients: S Pan, X Zhang, Y Pang; (IV) Collection and assembly of data: S Pan, R Ruan, Z Yan; (V) Data analysis and interpretation: S Pan, C Huang, X Zheng; (VI) Manuscript writing: All authors; (VII) Final approval of manuscript: All authors.

Correspondence to: Kaijin Guo, MD. Department of Orthopedics, The Affiliated Hospital of Xuzhou Medical University, 99 Huaihai Road West, Xuzhou 221006, China. Email: kaijinguo@163.com; Xin Zheng, MD. Department of Orthopedics, The Affiliated Hospital of Xuzhou Medical University, 99 Huaihai Road West, Xuzhou 221006, China; Department of Orthopedics, Zhujiang Hospital of Southern Medical University, 253 Gongye Avenue, Guangzhou 510282, China. Email: drzhengxin@163.com.

Background: Whole leg radiograph (WLR) is the gold standard in assessing lower limb alignment before total knee arthroplasty (TKA) although in practice, non-weight-bearing short knee radiographs (NWB SKRs) are used by most medical institutions. The objective of this study was to determine whether the femorotibial angle (FTA) could be used to evaluate lower limb alignment on limited NWB SKRs. We also investigated whether FTA alignment measurements on NWB SKRs and WLRs differed depending on the direction of knee deformity.

Methods: In all, 105 knees which underwent both NWB SKR and WLR were included. Measurement of hip-knee-ankle angle (HKA) was obtained through WLR, while the FTA was found using NWB SKR $\left(\mathrm{FTA}_{\mathrm{SKR}}\right)$ and WLR $\left(\mathrm{FTA}_{\mathrm{WLR}}\right)$. All knees were divided into three groups based on the HKA. The Kappa statistic was used to compare the agreement of categorical alignment variables between the HKA and FTA $_{\text {SKR }}$. The agreement of the measurements obtained from the two radiographs was made using BlandAltman plots and intraclass correlation coefficient (ICC). Pearson correlation coefficient and simple linear regression analysis were also conducted to evaluate the correlation between different angles.

Results: The agreement for categories of lower limb deformity was rated excellent (kappa $=0.804$, $\mathrm{P}<0.001)$. The Bland-Altman plot showed that the mean difference for the $\mathrm{FTA}_{\mathrm{SKR}}$ and $\mathrm{FTA}_{W L R}$ was $4.4^{\circ}$. There was an excellent correlation $(\mathrm{r}=0.861, \mathrm{P}<0.001)$ and good reliability $(\mathrm{ICC}, 0.607)$ between the FTA $\mathrm{SKR}$

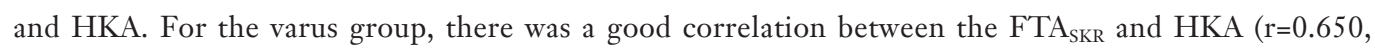
$\mathrm{P}<0.001)$; however, there were no significant correlations between the FTAS KR and HKA in the neutral $(\mathrm{r}=0.106, \mathrm{P}=0.543)$ and valgus groups $(\mathrm{r}=0.322, \mathrm{P}=0.102)$.

Conclusions: For outpatient follow-up, we found that the FTA on NWB SKRs is an acceptable means for classifying knee alignment (varus, neutral, or valgus). The measurement on NWB SKR also showed excellent correlation and good agreement with the HKA. For varus knees, NWB SKR measurements showed the best correlation with the HKA. However, for neutral and valgus knees, NWB SKR measurements were insufficient for conducting a reliable calculation and quantification of coronal alignment of the lower limb.

Keywords: Total knee arthroplasty (TKA); osteoarthritis (OA); coronal alignment; short knee radiograph; whole leg radiograph (WLR); femorotibial angle (FTA); mechanical axis 
Submitted Apr 13, 2021. Accepted for publication Sep 13, 2021.

doi: 10.21037/qims-21-400

View this article at: https://dx.doi.org/10.21037/qims-21-400

\section{Introduction}

Coronal alignment of the lower limbs plays a crucial role in evaluating the progression of osteoarthritis (OA) and in the postoperative assessment of total knee arthroplasty (TKA). Malalignment of the lower limbs can influence the development of knee disorders, such as patellofemoral joint complications and knee OA (1). Malalignment in TKA has been shown to increase the incidence of postoperative polyethylene wear, prosthesis loosening, infection, and periprosthetic fracture (2).

For TKA, weight-bearing whole leg radiograph (WB WLR) is the gold standard for evaluating the mechanical alignment (MA) of the lower limbs. Accurate assessment of MA is essential for the preoperative plan of osteotomy and postoperative assessment of implant position (3). However, this technique is time-consuming, requires special equipment, and involves significant radiation exposure (the pelvis is commonly exposed to a higher dose of X-ray radiation). The effective radiation from WLRs can be 73fold higher than that of short knee radiographs (SKRs) (4).

A standard knee radiograph is one of the primary methods to stage knee $\mathrm{OA}$ and predict the risk of joint space loss until operation (5). Additionally, it is used for many patients with knee disorders, and is ideal to investigate epidemiological studies. In practice, the femorotibial angle (FTA) is always measured on SKRs and is defined as the angle between the axis of the femur and tibia. Even if the hip and ankle joints are unable to be visualized in SKR, MA can still be calculated by putting FTA into the predictive equation (6-8). Although the application of FTA into the equation has not been widely accepted by the orthopedic community, it remains the best way to predict MA from a limited SKR.

van Raaij et al. (9) showed that the FTA on standing short knee films had a good correlation $(\mathrm{r}=0.65)$ with gold standard hip-knee-ankle angle (HKA) on WLR in 68 patients with symptomatic medial compartmental knee OA. However, in their study of the fully extended knee, Hunter et al. (10) did not find such a relationship between the FTA measured on standardized digital radiographs and the HKA on WB WLR. Furthermore, Sheey et al. (11) found that the relationship between the FTA and HKA can differ depending on the direction and magnitude of knee deformity.

Several experts $(12,13)$ have advocated the superiority of WLRs over SKRs. They have also deemed that the association between non-weight-bearing (NWB) SKRs and outlier measurements can result in the failure of osteotomy. In some cases, owing to equipment limitations or the inability to acquire WLRs in a timely manner, NWB SKR was reported to be the only technique available to assess lower limb alignment. Despite this, several studies $(3,5,14)$ have endeavored to establish a relationship between FTA by standing knee radiography and HKA on WLR (notably, there were limited reports regarding the NWB SKR before TKA). In our study, we aimed to investigate the application of NWB SKR before hospitalization, especially during the time of outpatient follow-up.

The objective of this study was to determine whether the FTA could be used to evaluate lower limb alignment on a limited NWB SKR both before operation and during outpatient follow-up. We also investigated whether FTA alignment measurements in NWB SKRs and WLRs differed depending on the direction of knee deformity.

\section{Methods}

This study was approved by the ethics board of the Affiliated Hospital of Xuzhou Medical University and was conducted in accordance with the Declaration of Helsinki (as revised in 2013). All patients gave written informed consent before participating. The inclusion criteria included patients who had both WLRs and NWB SKRs for reasons related to symptomatic knee OA. NWB SKRs were mainly employed to evaluate Kellgren-Lawrence (K-L) grades. The interval of the 2 radiographs was less than 3 months. Patients were excluded if they had a diagnosis other than primary knee OA, a significant bony deformity that restrained identification of the anatomical landmarks used for measurements, severe femoral lateral bowing, previous history of a homolateral lower limb operation (e.g., total hip arthroplasty, an operation for a femoral or tibial fracture, or high tibial osteotomy), knee flexion contraction $>20^{\circ}$, or a $\mathrm{K}-\mathrm{L}$ grade $<3$.

All images were measured by two experienced raters 


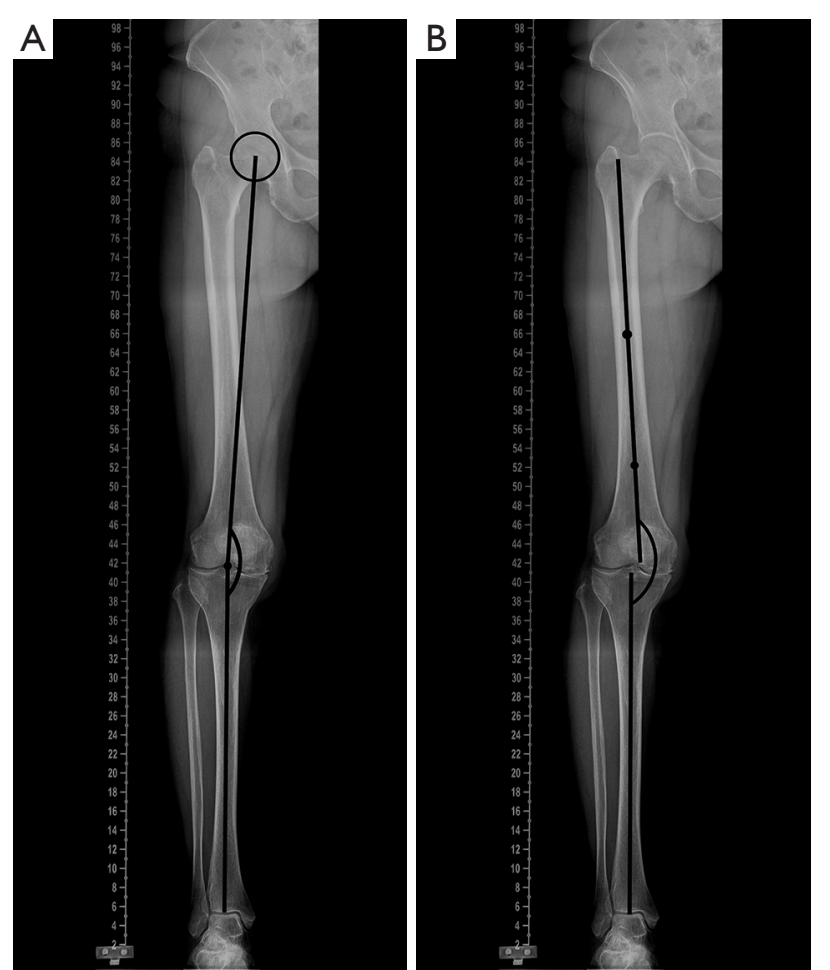

Figure 1 Measurement technique on WLR. (A) HKA; (B) FTA on WLR. HKA, hip-knee-ankle angle; FTA, femorotibial angle; WLR, whole leg radiograph.

with a 1-month interval between their measurements to confirm the HKA and FTA on WLR and another FTA on SKR. Considering the limited number of neutral and valgus knee cases in our database, we attempted to establish three groups that contained a balanced quantity of different groups. Finally, 105 selected knees retrospectively reviewed, with 50 cases randomly drawn from the varus group, 28 cases from the neutral group, and 27 cases from valgus group.

WLRs and NWB SKRs were taken by the DigitalDiagnost 3 radiograph system (Philips, Amsterdam, Netherlands). When WLR was taken, the patient stood upright in full knee extension with the back of the knee against the vertical cassette and the bilateral patella facing forward. When the NWB SKR was taken, the patient lay on the camera bed with the lower limbs kept straight and slightly internally rotated; the lower edge of the patella target was at the center of the detector, and the long axis of the limb was parallel to that of the detector. A picture archiving and communication system (PACS; GE Healthcare, Chicago, IL, USA) was used to transmit lower limb images and measure angles with its own protractor and cobb angle tool. The angles were measured down to the nearest $0.1^{\circ}$.

\section{Alignment measurements}

The HKA was defined as the intersecting angle of the femoral and tibial mechanical axis. The mechanical axis of the femur was drawn from the center of the femoral head to the apex of the femoral intercondylar notch. The center of the femoral head was identified by the concentric circle method. The line from the center of the tibial spine to the center of the talus established the mechanical axis of the tibia (15) (Figure 1A).

$\mathrm{FTA}_{W L R}$ was defined as the intersecting angle of the femoral and tibial anatomical axis on WLR. Two methods were used to define the femoral anatomical axis. First, a line was drawn from the mid-shaft point in the area where it bisects the femur's proximal-to-distal length and the center of the knee. In the metaphyseal region of the femur, however, this line is always on the lateral side of the femur. Therefore, in this study, the femoral anatomical axis was defined as the line between the point of the midshaft bisecting the proximal-to-distal length of the femur and located $10 \mathrm{~cm}$ above the femoral notch. The tibial anatomical axis was usually deemed equivalent to the tibial mechanical axis (16) (Figure 1B).

FTA $_{\text {SKR }}$ was defined as the intersecting angle of the femoral and tibial anatomical axis on SKR. The measurements were performed using a general technique and validated by Gielis et al. (17). To have a better reflection of femoral and tibial anatomical variations (18), we selected the more distant reference points of the femur and tibia (those which were closest to the edge of the image). The femoral anatomical axis was defined as the line between the mid-shaft close to the edge of the femur in the image, and the mid-shaft in the area where the metaphysis and epiphysis meet. For the tibial anatomic axis, a line was drawn from the mid-shaft close to the edge of the tibia in the image, and the mid-shaft in the area where the metaphysis and epiphysis meet (Figure 2).

All measured angles were subtracted from $180^{\circ}$. All knees were divided into 3 groups based on the HKA measured by preoperative $W L R$, with angle $<-3^{\circ}$ defined as the varus group, angle $\geq-3^{\circ}$, and $\leq 3^{\circ}$ defined as the neutral group, and all others defined as the valgus group. For SKR, an FTA $<2.4^{\circ}$ was considered varus, $2.4^{\circ}-7.2^{\circ}$ neutral, and $>7.2^{\circ}$ valgus (12). 


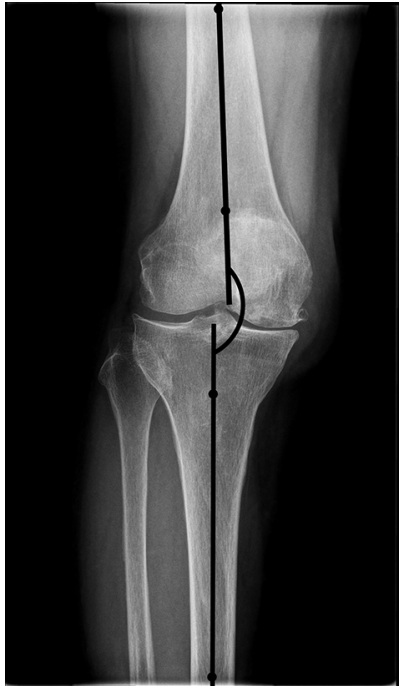

Figure 2 Measurement technique of FTA on NWB SKR. FTA, femorotibial angle; NWB SKR, non-weight-bearing short knee radiograph.

\section{Statistical analysis}

All data analyses were performed using SPSS statistical software version 25.0 (IBM, Armonk, NY, USA). The correlation between the $\mathrm{FTA}_{\mathrm{SKR}}$ and HKA was the primary outcome. A power analysis was performed with data from a previously published study by Sheey et al. (11), with at least an estimated 26 sample sizes for each subgroup needed to provide $80 \%$ power for correlation analysis, assuming the effect size index was 0.5 , with a 2 -sided $\alpha$ of 0.05 . Inter- and intraobserver reliabilities of measurements were evaluated by intraclass correlation coefficients (ICCs). Data were obtained by 2 experienced raters at 1 -month measurement intervals. The mean value of the 4 different measurements was used for analysis. An ICC value $>0.75$ indicated excellent reliability, 0.6 to 0.74 good, 0.4 to 0.59 moderate, and $<0.4$ poor. The Kappa statistic was used to compare the agreement of categorical alignment variables between $\mathrm{HKA}$ and $\mathrm{FTA}_{\mathrm{SKR}}$ (varus, neutral, and valgus). The agreement of $\mathrm{FTA}_{\mathrm{SKR}}$ and FTA $_{W L R}$ was compared by Bland-Altman plots. We plotted the magnitude (absolute value of the mean difference) rather than the direction of the difference in the 2 methods to minimize the deviation, as the positive values tend to cancel out the negative. The correlation between angles was determined by Pearson's correlation coefficient. Simple linear regression analysis was conducted to develop regression equations for identifying the correlations between angles for statistically significant relationships. Correlation coefficients of $0.5-0.75$
Table 1 Agreement for categories of lower limb deformity (kappa $=0.804, \mathrm{P}<0.001$ )

\begin{tabular}{lcccc}
\hline \multirow{2}{*}{$\begin{array}{l}\text { HKA measured } \\
\text { deformity }\end{array}$} & \multicolumn{2}{c}{ NWB SKR measured deformity } & \multirow{2}{*}{ Total } \\
\cline { 2 - 4 } & Varus & Neutral & Valgus & \\
\hline Varus & 48 & 2 & 0 & 50 \\
Neutral & 3 & 23 & 2 & 28 \\
Valgus & 1 & 5 & 21 & 27 \\
Total & 52 & 30 & 23 & 105 \\
\hline
\end{tabular}

NWB SKR, non-weight-bearing short knee radiograph; HKA, hip-knee-ankle angle.

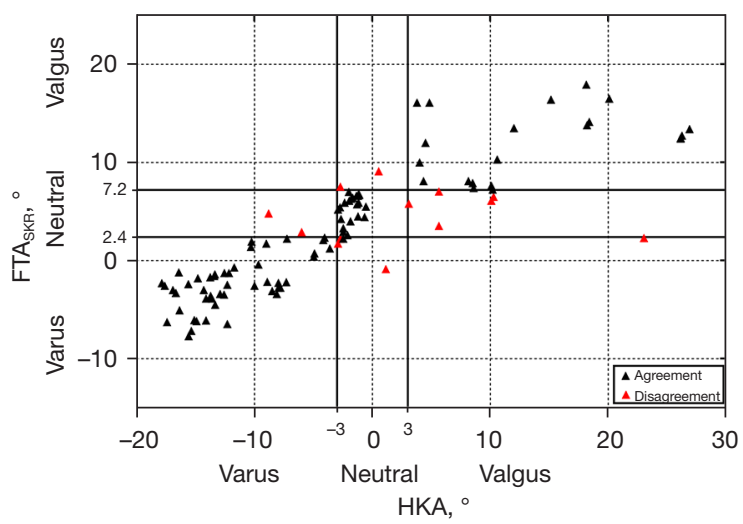

Figure 3 This scatterplot depicts the FTA $_{\mathrm{SKR}}$ and HKA. The black triangles represent knees that were classified as varus, neutral, or valgus on both SKRs and WLRs, and the red triangles represent knees whose classifications disagreed on the 2 radiographs. FTA $_{\mathrm{SKR}}$, femorotibial angle on short knee radiograph; HKA, hip-knee-ankle angle; SKR, short knee radiograph; WLR, whole leg radiograph.

were deemed good, and values $>0.75$ were deemed excellent. ICCs (2-way random effects model, absolute agreement) were also used to establish the reliability between the different methods. $\mathrm{P}$ values $<0.05$ were considered significant.

\section{Results}

The mean age of patients was $66.7 \pm 8.1$ years (range, 45-83 years), and their mean body mass index (BMI) was $26.7 \pm 3.6 \mathrm{~kg} / \mathrm{m}^{2}$ (range, $18.3-36.4 \mathrm{~kg} / \mathrm{m}^{2}$ ). There were 23 males and 58 left knees.

For the 3 parameters, ICCs of inter- and intra-observer reliabilities were both greater than 0.90 , which indicated excellent reliability.

As shown in Table 1 and Figure 3, the agreement for 


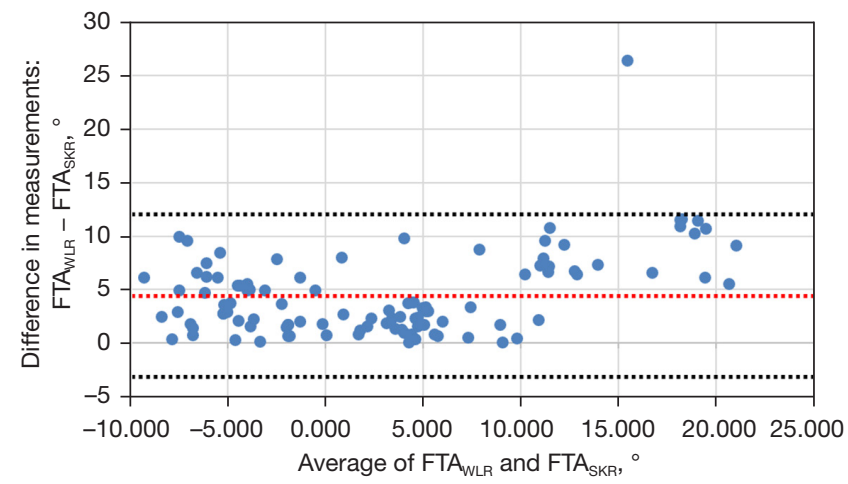

Figure 4 Bland-Altman plot for $\mathrm{FTA}_{W L R}$ and $\mathrm{FTA}_{\mathrm{SKR}}$. The red dotted line represents the mean difference, and the dotted lines show $95 \%$ limits of agreement (the difference in 2 measurements was considered the absolute value). $\mathrm{FTA}_{\mathrm{WLR}}$, femorotibial angle on whole leg radiograph; FTA $_{\mathrm{SKR}}$, femorotibial angle on short knee radiograph.

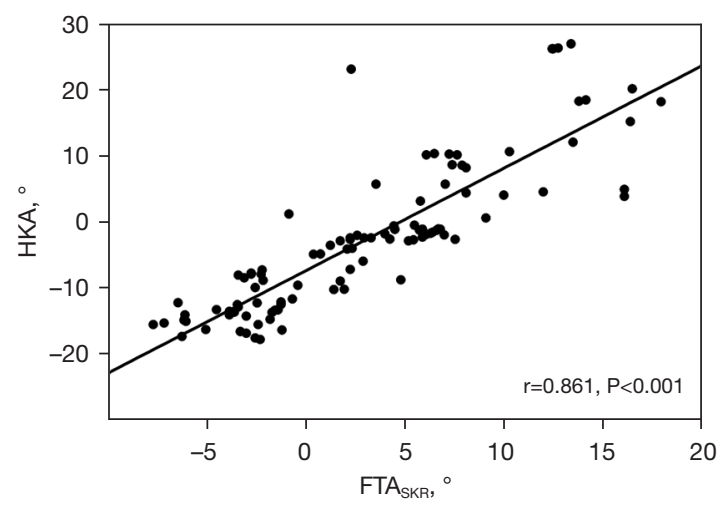

Figure 5 Scattered plot with linear regression showing the overall correlation between HKA and FTA $_{\mathrm{SKR}}$. HKA, hip-knee-ankle angle; FTA $_{\mathrm{SKR}}$, femorotibial angle on short knee radiograph.

categories of lower limb deformity was excellent (kappa $=0.804, \mathrm{P}<0.001)$. Assuming that the HKA measured on WLR gave the correct deformity, $87.6 \%$ of knees were categorized correctly by the $\mathrm{FTA}_{\mathrm{SKR}}$. Only $4 \%$ of varus knees were categorized incorrectly by the $\mathrm{FTA}_{\mathrm{SKR}}$. However, $17.8 \%$ of neutral and $22.8 \%$ of valgus knees were categorized incorrectly by the $\mathrm{FTA}_{\mathrm{SKR}}$.

The Bland-Altman plot in Figure 4 shows that the mean difference for the $\mathrm{FTA}_{\mathrm{SKR}}$ and $\mathrm{FTA}_{W L R}$ was $4.4^{\circ}$ with a $95 \%$ upper- and lower-level agreement of $-3.2^{\circ}$ and $12.0^{\circ}$, respectively.

There was an excellent correlation between FTA $_{\mathrm{SKR}}$

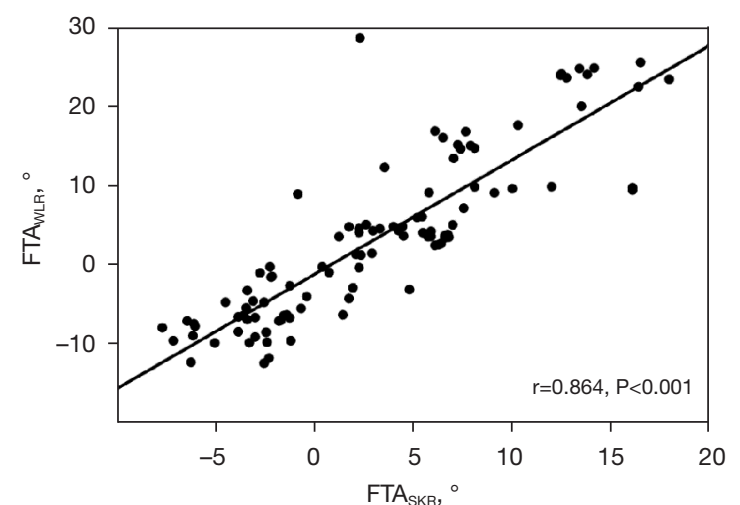

Figure 6 Scattered plot with linear regression showing the overall correlation between $\mathrm{FTA}_{\mathrm{WLR}}$ and $\mathrm{FTA}_{\mathrm{SKR}}$. $\mathrm{FTA}_{\mathrm{WLR}}$, femorotibial angle on whole leg radiograph; FTA $_{\mathrm{SKR}}$, femorotibial angle on short knee radiograph.

and HKA ( $\mathrm{r}=0.861, \mathrm{P}<0.001$; Figure 5). According to simple linear regression analysis, $74.1 \%$ of the variation in the HKA could be explained by $\mathrm{FTA}_{\mathrm{SKR}}$. The reliability between FTA $_{\text {SKR }}$ and HKA was good (ICC, 0.607). There was also a strong positive correlation between the $\mathrm{FTA}_{\mathrm{SKR}}$ and $\mathrm{FTA}_{W L R}(\mathrm{r}=0.864, \mathrm{P}<0.001$; Figure 6). The reliability between FTA $\mathrm{FKR}_{\mathrm{SKR}}$ and $\mathrm{FTA}_{\mathrm{WLR}}$ was excellent (ICC, 0.763).

Further analysis of different knee direction deformities was performed. For the varus group, there was a good correlation between $\mathrm{FTA}_{\mathrm{SKR}}$ and HKA $(\mathrm{r}=0.650, \mathrm{P}<0.001)$, and $42.2 \%$ of the variation in the HKA was explained by FTA $_{\text {SKR }}$. However, there were no significant correlations between the $\mathrm{FTA}_{\mathrm{SKR}}$ and HKA in both the neutral $(\mathrm{r}=0.106$, $\mathrm{P}=0.543)$ and valgus $(\mathrm{r}=0.322, \mathrm{P}=0.102)$ groups. Their regression coefficients were not statistically significant (Table 2).

\section{Discussion}

The most important finding of the present study was that the agreement for categories of lower limb deformity was excellent. Therefore, we believe FTA on NWB SKR has the potential to be used for classifying knee alignment (varus, neutral, or valgus). Our hypothesis that the relationship between the FTA by NWB SKR and the HKA would differ depending on the direction of knee deformity was thus verified.

On different occasions, comparisons have been made between $\mathrm{X}$-ray measurements of the whole leg versus just the knee. van Raaij et al. (9) found that the FTA measurement using the mid-diaphyseal lines of the femur 
Table 2 Results from simple linear regression analysis of different knee direction deformities

\begin{tabular}{|c|c|c|c|c|c|c|}
\hline Group & $\mathrm{R}^{2}$ & Pearson coefficient $(r)$ & & Beta & Standard error & $\mathrm{P}$ \\
\hline Complete dataset & & & $\mathrm{FTA}_{\mathrm{SKR}}$ & 1.550 & 0.090 & $<0.001$ \\
\hline \multirow[t]{2}{*}{ Varus } & $42.2 \%$ & 0.650 & Constant & -9.707 & 0.543 & $<0.001$ \\
\hline & & & $\mathrm{FTA}_{\mathrm{SKR}}$ & 0.918 & 0.155 & $<0.001$ \\
\hline Neutral & & & $\mathrm{FTA}_{\mathrm{SKR}}$ & 0.049 & 0.091 & 0.543 \\
\hline \multirow[t]{2}{*}{ Valgus } & $10.4 \%$ & 0.322 & Constant & 6.398 & 3.993 & 0.122 \\
\hline & & & $\mathrm{FTA}_{\mathrm{SKR}}$ & 0.596 & 0.351 & 0.102 \\
\hline
\end{tabular}

Independent variable: FTA $_{\mathrm{SKR}}$. FTA $\mathrm{SKR}_{\mathrm{SR}}$, femorotibial angle on short knee radiograph.

and tibia on standing SKR had a good correlation with MA, and may be beneficial for defining the precise entry point for intramedullary alignment guides when WLR is not readily available. However, Stickley et al. (8) insisted that standing SKR is inadequate to determine MA and should not be used to template the angle of a distal femoral cut before operation. Oh et al. (19) also concluded standard SKRs are deficient in evaluating coronal alignment after TKA, particularly in knees with femoral bowing. Chang et al. (18) showed that the presence of lateral bowing of the femur is a major cause of MA to TFA variations. To minimize the effect of femoral bowing and other anatomical variations, we selected the more distant reference points of the femur and tibia (those closest to the edge of the image). Skyttä et al. (20) reported that the standing SKR is a valid alternative to the WLR for estimating coronal alignment in routine follow-up after TKA. These comparisons of $\mathrm{X}$-ray measurements focused on the WLR and standing knee radiograph, but there are limited reports regarding the NWB SKR before TKA. To our knowledge, our study is the first to investigate both the classification and relationship of measurements between WLR and NWB SKR in patients with OA before operation. Combined with the high incidence of varus knee in China, we concluded that SKR has the potential to classify knee alignment (varus, neutral, or valgus) during outpatient follow-up but found its usage for preoperative templating was limited due its calculations of the neutral and valgus knees. In other words, our research defined the application scope of NWB SKR.

Several studies have reported on the difference and relationship between lower limb radiographs with WB and NWB positions. Shin et al. (21) analyzed the accuracy of NWB and WB radiographs before a medial open-wedge high tibial osteotomy. They found the mean HKA in the WB position $\left(8.54^{\circ} \pm 3.38^{\circ}\right)$ was significantly higher than that in the NWB position $\left(6.65^{\circ} \pm 2.71^{\circ}\right)$. Meanwhile, Kim et al. (22) found the HKA in the preoperative WB radiograph was significantly higher than that in the NWB radiograph $\left(10.9^{\circ} \pm 5.2^{\circ}\right.$ vs. $\left.7.7^{\circ} \pm 6.9^{\circ}, \mathrm{P}<0.001\right)$. They also showed the agreement between the FTA on SKR and WLR in the WB position was greater than that in the NWB position (ICC $0.878 v$ s. 0.657). Additionally, the FTA was often measured on SKR using lines drawn from the knee to a point approximately $10 \mathrm{~cm}$ proximal to the center of the knee (17); however, the location the point for the bone shaft also might alter the correlation of FTA to HKA. Sheey's et al. (11) compared different versions of FTA and HKA using different reference points and concluded that using shorter FTA shaft lengths to evaluate HKA modestly weakened the correlation of the 2 measurements.

Classifying knees in different directions plays a major role in load distribution of the knee and is a significant determination of OA progression (15). In patients with primary knee $\mathrm{OA}$, it has been reported that varus alignment increases the risk of medial OA progression, while valgus alignment increases the risk of lateral OA progression. Malalignment of more than $5^{\circ}$ (either in the varus or valgus direction) has been shown to be correlated with more functional deterioration compared with a knee alignment of $5^{\circ}$ or less (15). Brouwer et al. (23) also found that an advancing degree of varus malalignment was associated with the progression and development of knee OA although this finding was only applicable to overweight persons. Furthermore, they found knee alignment to be a predictor of the rotational geometry of the distal femur in $\mathrm{OA}$ patients, with patients with valgus malalignment showing 
hypoplasia of the lateral femoral condyle, potentially affecting the dynamic alignment of the lower extremity after TKA (24).

van Raaij et al. (9) compared preoperative radiographs of 68 patients with varus $\mathrm{OA}$ of the knee. Based on the FTA measured on short knee films, only $3 \%$ of knees were misinterpreted as having valgus deformities. In Colebatch et al.'s study (25), 10\% of knees were coded incorrectly as varus knees on anteroposterior (AP) knee radiographs, but were correctly reclassified as neutral on full-limb radiographs (kappa $=0.65-0.74$ ). Our agreement for categories of lower limb deformity was greater than Colebatch et al.'s (kappa $=0.804 ; \mathrm{P}<0.001)$. Assuming that the HKA measured on WLR gave the correct deformity, $87.6 \%$ of knees were categorized correctly by the $\mathrm{FTA}_{\mathrm{SKR}}$ in our study. When the $12.4 \%$ inconsistent cases were considered, it was found that only $4 \%$ of the varus cases were classified wrongly by the $\mathrm{FTA}_{\mathrm{SKR}}$. Misdiagnosis was mainly caused by neutral and valgus knees. As Chinese patients with OA have a strong predominance of varus deformity, we deemed $\mathrm{FTA}_{\mathrm{SKR}}$ to be an acceptable method for classifying knee alignment, especially during outpatient follow-up.

In this study, we found a strong positive correlation between FTA $_{\text {SKR }}$ and HKA (r=0.861). Notably, our Pearson coefficient results were higher than those in other studies $(6,7,9)$. We believe that the more distant reference points of the femur and tibia which were close to the edge of the image could reduce the influence of femoral and tibial anatomical variations and promote the ability of FTA $_{\mathrm{SKR}}$ to assess coronal alignment (18). The findings of Sheehy et al. (11) and Hinman et al. (26) were comparable with our own (both $\mathrm{r}=0.88$ ). In their study, the anatomical axis of the knee was measured on the full-leg radiographs which could reduce the impact of shooting angle and position when taking radiographs. In addition, in Hinman et al.'s study (26), the participants all had medial compartmental knee OA. As seen from our results, varus knees have a stronger correlation between FTA $_{\mathrm{SKR}}$ and HKA. In our opinion, the correlation between the two measurements demonstrates a better value if there is a fixed deviation between them. For this reason, we also evaluated the reliability between FTA $_{\text {SKR }}$ and HKA. The agreement between the 2 methods was not found to be excellent, but still good (ICC, 0.607).

The relationship between FTA $_{\text {SKR }}$ and HKA is attenuated when cases are categorized by the direction of the knee deformity. Sheehy et al. (11) asserted that the relationship between the two measurements depends on the direction of the knee deformity. In our study, the correlation coefficient for varus knees was 0.650 , which is close to Sheehy's $(r=0.45)$. However, Unal et al.'s findings concerning the relationship to the varus knee were inconsistent with our own, and they found no correlation between the two methods for varus knees (6). Furthermore, we did not find any correlation between the two methods for neutral and valgus knees. A lower correlation was also found as the HKA increased. Perhaps OA patients with neutral and valgus knees have a more severe extra-articular deformity, and identifying anatomic variations of the whole femur and tibia is more easily achieved by WLR.

Regarding the relation between the FTA $_{\text {SKR }}$ and $\mathrm{FTA}_{W L R}$, Kraus et al. (7) reported a good correlation between the two alignments measured from fixed-flexion radiographs and full-length radiographs $(\mathrm{r}=0.73)$. Unal et al. (6) also found a very strong positive correlation between the anatomical axis on a short knee anteroposterior $\mathrm{X}$-ray, which was derived by cropping WLR and the orthoroentgenogram anatomical axis $(r=0.83)$. The correlations in our study were similar to those reported by Unal et al. $(\mathrm{r}=0.864)$. The reliability of both the $\mathrm{FTA}_{\mathrm{SKR}}$ and FTA $_{\text {WLR }}$ in the present study were also comparable with those reported by Matos et al. (27) and Alzahrani et al. (3), which all indicated excellent reliability. Matos et al. (27) observed a strongly significant agreement (ICC $>0.88$ as a minimum) between the anatomical axis using SKR and WLR both with weight borne on 2 feet. Alzahrani et al. (3) performed a reliability analysis of coronal alignment between WB SKR and WLR in preoperative radiographs. They found that the FTA and medial proximal tibial angle had excellent correlation, with ICCs of 0.84 and 0.83 .

The following details the limitations of our study. (I) It was a retrospective study, and further prospective studies are required to probe the validity of the relationships analyzed. (II) All patients had severe $\mathrm{AO}$ with a $\mathrm{K}-\mathrm{L}$ grade greater than 3, and so no mild OA cases were included. However, considering that patients with mild OA usually do not require surgical treatment, they do not usually present to a clinic until the end stage of OA. Furthermore, only patients with end stage OA can undergo WLR examination, which is useful for preoperative guidance and planning. (III) The WLR and SKR were not always taken the same day, and thus the consistency of the patients' lower limb position could not be guaranteed. (IV) We did not calculate the variability between the two angles separately for male and female. (V) Preoperative computed tomography (CT) could provide us with more information about the alignment 
of the lower limb (i.e., not only coronal alignment) (28). However, considering the higher cost and radiation of CT, it is not suitable for routine examination before operation. Considering these limitations, further studies are required to investigate the application of SKR before TKA.

\section{Conclusions}

For outpatient follow-up, FTA on NWB SKR is an acceptable method for classifying knee alignment (varus, neutral, or valgus). The measurement on NWB SKR showed excellent correlation and good agreement with the HKA. For varus knees, NWB SKR measurements showed the best correlation with the HKA. However, for neutral and valgus knees, NWB SKR measurements were insufficient for conducting a reliable calculation and quantification of coronal alignment of the lower limb.

\section{Acknowledgments}

Funding: This study was supported by the National Natural Science Foundation of China (No. 81902244), the Natural Science Foundation of Jiangsu Province (No. BK20201154), the Jiangsu Provincial Medical Youth Talent (No. QNRC2016801), and the Xuzhou Science and Technology Innovation Project (No. KC19063).

\section{Footnote}

Conflicts of Interest: All authors have completed the ICMJE uniform disclosure form (available at https://dx.doi. org/10.21037/qims-21-400). The authors have no conflicts of interest to declare.

Ethical Statement: The authors are accountable for all aspects of the work in ensuring that questions related to the accuracy or integrity of any part of the work are appropriately investigated and resolved. The study was conducted in accordance with the Declaration of Helsinki (as revised in 2013). The study was approved by the ethics board of our hospital, and informed consent was taken from all individual participants.

Open Access Statement: This is an Open Access article distributed in accordance with the Creative Commons Attribution-NonCommercial-NoDerivs 4.0 International License (CC BY-NC-ND 4.0), which permits the noncommercial replication and distribution of the article with the strict proviso that no changes or edits are made and the original work is properly cited (including links to both the formal publication through the relevant DOI and the license). See: https://creativecommons.org/licenses/by-nc-nd/4.0/.

\section{References}

1. Folinais D, Thelen P, Delin C, Radier C, Catonne Y, Lazennec JY. Measuring femoral and rotational alignment: EOS system versus computed tomography. Orthop Traumatol Surg Res 2013;99:509-16.

2. Sharkey PF, Lichstein PM, Shen C, Tokarski AT, Parvizi J. Why are total knee arthroplasties failing today-has anything changed after 10 years? J Arthroplasty 2014;29:1774-8.

3. Alzahrani MM, Wood TJ, Somerville LE, Howard JL, Lanting BA, Vasarhelyi EM. Correlation of Short Knee Radiographs and Full-length Radiographs in Patients Undergoing Total Knee Arthroplasty. J Am Acad Orthop Surg 2019;27:e516-21.

4. Hart D, Wall BF. Radiation exposure of the UK population from medical and dental X-ray examinations. Chilton, NRPB-R289 (2002). Available online: https://webarchive. nationalarchives.gov.uk/ukgwa/20100303233542/http:// www.hpa.org.uk/webw/HPAweb\&HPAwebStandard/ HPAweb_C/1247816602523?p=1158945066506

5. Felson DT, Cooke TD, Niu J, Goggins J, Choi J, Yu J, Nevitt MC; OAI Investigators Group. Can anatomic alignment measured from a knee radiograph substitute for mechanical alignment from full limb films? Osteoarthritis Cartilage 2009;17:1448-52.

6. Unal M, Ercan S, Budeyri A, Toprak U, Şalkaci A. Anatomical axis validation of lower extremity for different deformities: A radiological study. SAGE Open Med 2020;8:2050312120923822.

7. Kraus VB, Vail TP, Worrell T, McDaniel G. A comparative assessment of alignment angle of the knee by radiographic and physical examination methods. Arthritis Rheum 2005;52:1730-5.

8. Stickley CD, Wages JJ, Hetzler RK, Andrews SN, Nakasone CK. Standard Radiographs Are Not Sufficient for Assessing Knee Mechanical Axis in Patients With Advanced Osteoarthritis. J Arthroplasty 2017;32:1013-7.

9. van Raaij TM, Brouwer RW, Reijman M, Bierma-Zeinstra SM, Verhaar JA. Conventional knee films hamper accurate knee alignment determination in patients with varus osteoarthritis of the knee. Knee 2009;16:109-11.

10. Hunter DJ, Niu J, Felson DT, Harvey WF, Gross KD, 
McCree P, Aliabadi P, Sack B, Zhang Y. Knee alignment does not predict incident osteoarthritis: the Framingham osteoarthritis study. Arthritis Rheum 2007;56:1212-8.

11. Sheehy L, Felson D, Zhang Y, Niu J, Lam YM, Segal N, Lynch J, Cooke TD. Does measurement of the anatomic axis consistently predict hip-knee-ankle angle (HKA) for knee alignment studies in osteoarthritis? Analysis of long limb radiographs from the multicenter osteoarthritis (MOST) study. Osteoarthritis Cartilage 2011;19:58-64.

12. Park A, Stambough JB, Nunley RM, Barrack RL, Nam D. The Inadequacy of Short Knee Radiographs in Evaluating Coronal Alignment After Total Knee Arthroplasty. J Arthroplasty 2016;31:878-82.

13. Borton Z, Shivji F, Eyre-Brook AI, Wilson A, Yasen S. Non-weightbearing imaging and standard knee radiographs are inferior to formal alignment radiographs for calculating coronal alignment of the knee. Radiography (Lond) 2021;27:260-5.

14. Abu-Rajab RB, Deakin AH, Kandasami M, McGlynn J, Picard F, Kinninmonth AW. Hip-Knee-Ankle Radiographs Are More Appropriate for Assessment of Post-Operative Mechanical Alignment of Total Knee Arthroplasties than Standard AP Knee Radiographs. J Arthroplasty 2015;30:695-700.

15. Sharma L, Song J, Felson DT, Cahue S, Shamiyeh E, Dunlop DD. The role of knee alignment in disease progression and functional decline in knee osteoarthritis. JAMA 2001;286:188-95.

16. Moreland JR, Bassett LW, Hanker GJ. Radiographic analysis of the axial alignment of the lower extremity. J Bone Joint Surg Am 1987;69:745-9.

17. Gielis WP, Rayegan H, Arbabi V, Ahmadi Brooghani SY, Lindner C, Cootes TF, de Jong PA, Weinans H, Custers RJH. Predicting the mechanical hip-knee-ankle angle accurately from standard knee radiographs: a crossvalidation experiment in 100 patients. Acta Orthop 2020;91:732-7.

18. Chang CB, Choi JY, Koh IJ, Seo ES, Seong SC, Kim TK. What should be considered in using standard knee radiographs to estimate mechanical alignment of the knee? Osteoarthritis Cartilage 2010;18:530-8.

19. Oh SM, Bin SI, Kim JY, Lee BS, Kim JM. Short knee radiographs can be inadequate for estimating TKA alignment in knees with bowing. Knee Surg Relat Res 2020;32:9.

20. Skyttä ET, Lohman M, Tallroth K, Remes V. Comparison of standard anteroposterior knee and hip-to-ankle radiographs in determining the lower limb and implant alignment after total knee arthroplasty. Scand J Surg 2009;98:250-3.

21. Shin KH, Jung JK, Nam JJ, Jang KM, Han SB. Preoperative Supine Radiographs Are More Accurate Than Standing Radiographs for Preoperative Planning in Medial Open-Wedge High Tibial Osteotomy. Arthroscopy 2020;36:1655-64.

22. Kim SH, Park YB, Song MK, Lim JW, Lee HJ. Reliability and Validity of the Femorotibial Mechanical Axis Angle in Primary Total Knee Arthroplasty: Navigation versus Weight Bearing or Supine Whole Leg Radiographs. Knee Surg Relat Res 2018;30:326-33.

23. Brouwer GM, van Tol AW, Bergink AP, Belo JN, Bernsen RM, Reijman M, Pols HA, Bierma-Zeinstra SM. Association between valgus and varus alignment and the development and progression of radiographic osteoarthritis of the knee. Arthritis Rheum 2007;56:1204-11.

24. Chang MJ, Jeong HJ, Kang SB, Chang CB, Yoon C, Shin JY. Relationship Between Coronal Alignment and Rotational Profile of Lower Extremity in Patients With Knee Osteoarthritis. J Arthroplasty 2018;33:3773-7.

25. Colebatch AN, Hart DJ, Zhai G, Williams FM, Spector TD, Arden NK. Effective measurement of knee alignment using AP knee radiographs. Knee 2009;16:42-5.

26. Hinman RS, May RL, Crossley KM. Is there an alternative to the full-leg radiograph for determining knee joint alignment in osteoarthritis? Arthritis Rheum 2006;55:306-13.

27. Matos LF, Giordano M, Cardoso GN, Farias RB, E Albuquerque RP. Comparative radiographic analysis on the anatomical axis in knee osteoarthritis cases: inter and intraobserver evaluation. Rev Bras Ortop 2015;50:283-9.

28. Campanelli V, Lozano R, Akhlaghpour H, Brar AS, Maislin D, Nedopil AJ, Zuhars J. Implant placement accuracy in total knee arthroplasty: validation of a CTbased measurement technique. Quant Imaging Med Surg 2020;10:475-84.

Cite this article as: Pan S, Huang C, Zhang X, Ruan R, Yan Z, Li Z, Pang Y, Guo K, Zheng X. Non-weight-bearing short knee radiographs to evaluate coronal alignment before total knee arthroplasty. Quant Imaging Med Surg 2022;12(2):12141222. doi: 10.21037/qims-21-400 